\title{
MICROECONOMÍA DE LA PRODUCCIÓN DE GANADO VACUNO DE CARNE EN EL VALLE MEDIO DEL RIO SINÚ (MONTERÍA - COLOMBIA): UN ESTUDIO DE CASO*
}

\author{
OMAR CASTILLO NUÑEZ ${ }^{* *}$, MARÍA KERGUELEN MACEA*** \& MARÍA NEGRETTE GUZMÁN"**** \\ UNIVERSIDAD DE CORDOBA
}

Recibido/ Received/ Recebido: 25/03/15 - Aceptado/ Accepted / Aprovado: 19/06/15

\begin{abstract}
Resumen
Este artículo tiene como objetivo contrastar empíricamente aspectos de la teoría microeconómica de la producción aplicada a una unidad empresarial de ganado vacuno cebado en pie en Montería (Colombia) para los años 2005-2013. Para tal fin se describe la técnica de producción utilizada, se evalúan los resultados técnicos y económicos, se modela la función de costo total, y se construye una función de oferta-precio mediante técnicas econométricas. Los resultados aportan evidencia empírica adicional acerca de que el uso de técnicas intensivas en alimentación con pasturas naturales mejoradas, suplementos y cuidado de la salud animal, permiten obtener resultados de producción y productividad física que se traducen en excedentes económicos positivos. La función de costo total mantiene una relación positiva con el nivel de producto y el precio de los insumos. El costo marginal es creciente y se intersecta con la función de costo medio mínimo en un nivel de producción de 33.529 kilogramos, correspondiente a un costo mínimo de $\$ 1.410$ por kilogramo. Consistente con la función de costo marginal creciente, la función de oferta-precio tiene pendiente positiva para señalar que los productores responden positivamente a los incentivos de precio del mercado. Palabras clave: Ganado cebado; Técnica de producción; Costos; Margen bruto; Oferta-precio.
\end{abstract}

\section{MICROECONOMICS OF BEEF CATTLE PRODUCTION IN THE SINÚ'S RIVER MIDDLE VALLEY (MONTERÍA - COLOMBIA): A CASE STUDY}

\begin{abstract}
This article aims to contrast empirically aspects of microeconomic theory of production applied to a business unit of fattened cattle standing in Monteria (Colombia) for the years 2005-2013. To this purpose the production technique used is described, the technical and economic results are evaluated, the total cost function is modeled, and a price supply function is built using econometric techniques. The results provide further empirical evidence on the use of intensive techniques in
\end{abstract}

\footnotetext{
Artículo resultado del proyecto de extensión colaborativo "Seguimiento a indicadores técnicos y económicos ganaderos", realizado entre el Observatorio de precios y costos agrarios (OPCA) y la empresa Lituania en el año 2014.

** Doctor en Economía Agraria, Universidad Politécnica de Madrid, España. Profesor titular, Universidad de Córdoba. Grupo Observatorio de precios y costos agrarios del noroccidente del caribe. Dirección postal: Carrera 6 No. 76-103 Montería - Córdoba (Colombia) Código Postal: 230002. Teléfono: +57(4)7860151. Correo electrónico: casnuom@gmail.com.

*** Economista, Universidad Sergio Arboleda, Bogotá.

****** Economista, Universidad Sergio Arboleda, Bogotá.
} 
feeding with improved natural pastures, supplements and animal health care, can deliver results in physical productivity and production that result in positive economic surplus. The total cost function maintains a positive relationship with the level of product and input prices. The marginal cost is increasing and intersects with the minimum average cost function in a production level of 33,529 kilograms, corresponding to a minimum cost of $\$ 1,410$ per kilogram. Consistent with the function of increasing marginal cost, the money supply function has positive slope to indicate that producers respond positively to the incentives of market price.

Keywords: Fed cattle; Production technique; Costs; Gross margin; Supply-Price.

\title{
MICROECONOMIA DA PRODUÇÃO DE GADO VACUNO DE CARNE NO VALE MÉDIO DO RIO SINÚ (MONTERÍA - COLÔMBIA): UM ESTUDO DE CASO
}

\begin{abstract}
Resumo
Este artigo tem como objetivo contrastar empiricamente aspectos da teoria microeconômica da produção aplicada a uma unidade empresarial de gado vacuno cevado em pé em Montería (Colômbia) para os anos 2005-2013. Para tal fim descreve-se a técnica de produção utilizada, avaliam-se os resultados técnicos e econômicos, modela-se a função de custo total, e constrói-se uma função de oferta-preço mediante técnicas econométricas. Os resultados geram evidência empírica adicional a respeito de que o uso de técnicas intensivas em alimentação com pastos naturais melhorados, suplementos e cuidado da saúde animal, permitem obter resultados de produção e produtividade física que se traduzem em excedentes econômicos positivos. A função de custo total mantém uma relação positiva com o nível de produto e o preço dos insumos. O custo marginal é crescente e se intersecta com a função de custo médio mínimo em um nível de produção de 33.529 quilogramas, correspondente a um custo mínimo de $\$ 1.410$ por quilograma. Consistente com a função de custo marginal crescente, a função de oferta-preço tem pendente positiva para assinalar que os produtores respondem positivamente aos incentivos de preço do mercado.
\end{abstract}

Palavras chave: Gado cevado; Técnica de produção; Custos; Margem bruta; Oferece-preço.

Castillo, O., Kerguelen, M. \& Negrette, M. (2015). Microeconomía de la producción de ganado vacuno de carne en el valle medio del rio sinú (Montería - Colombia): un estudio de caso. En: Revista de la Facultad de Ciencias Económicas de la Universidad Militar Nueva Granada. rev.fac.cienc.econ, XXIII (2), DOI: $h$ ttp://dx.doi.org/10.18359/rfce.1611.

JEL: D22, D24.

\section{Introducción}

El departamento de Córdoba, Colombia, es desde el punto de vista de la intención del productor de ganado vacuno, un departamento productor de carne. En efecto, según el Departamento Administrativo Nacional de Estadísticas (DANE, 2013), del total de las 2.086.159 cabezas existentes, 1.072.199 de ellas están orientadas a la producción de carne, es decir, el 51,4\%.
La actividad de la ceba es un negocio ganadero cuyo objetivo es el de producir ganados machos gordos para la venta y sacrificio mediante distintas técnicas de alimentación y pastoreo de los animales de modo que se obtenga un peso físico ideal (entre 450 y 480 kilos) en el menor tiempo posible dentro de los límites físicos de crecimiento de un animal vivo. A esta actividad de la ceba o de engorde de ganado vacuno se dedica la empresa ganadera Lituania, ubicada a $45 \mathrm{~km}$, aproximadamente, de la ciudad de Montería, 
Córdoba, Colombia. Situada al frente del rio Sinú, es un terreno de 84 hectáreas, sembrada en pastos de Angleton, Climacuna, Alemana y Braquiaria para alimentación de animales de raza cebú como Angus, Brahmán y Romosinuano, básicamente.

Como todo proceso productivo, en esta empresa se implementan actividades técnicas relacionadas con la forma como se combinan los distintos factores productivos e insumos en la actividad ganadera: tierra, en el sentido de recursos naturales y ambientales como suelos, luz del sol, clima, agua, etc.; la fuerza de trabajo, el capital (animales en producción, maquinas, equipos instalaciones e infraestructuras físicas en general) y la capacidad empresarial. Pero también, muy relacionados con el uso de la tecnología, están los aspectos económicos relacionados con los costos en que se incurre por el uso de los factores productivos, la capacidad de la empresa de responder con su producto a los requerimientos del mercado, y, obviamente, la evaluación de su desempeño económico como negocio.

En este orden de ideas, el presente artículo tiene como objetivo analizar el proceso de producción de carne de ganado vacuno cebado en pie en una unidad productiva empresarial en el departamento de Córdoba, Colombia, mediante la descripción de la técnica usada, la evaluación del desempeño económico, y el establecimiento de relaciones entre la producción, los costos y la oferta entre los años 2005-2014.

Desde el punto de vista teórico, esta investigación permite contrastar aspectos de la teoría Microeconómica de la producción con información empírica procedente de una unidad productiva concreta dedicada a la actividad de ceba ganadera bovina. En este sentido es un ejercicio de Economía aplicada a empresas del sector agrario, válido en una región donde las actividades agrarias tienen una presencia preponderante en la economía rural y general.

En lo práctico, contribuye a identificar experiencias regionales de desarrollo empresarial en una actividad de la que se ha dicho tiene problemas de baja rentabilidad, ineficiencias en el uso de los recursos, lenta adopción de tecnología, limitado emprendimiento, e insuficiencias de base de datos.
En lo metodológico, su importancia se ve reflejada en el tratamiento y análisis de la información recopilada, al usar herramientas estadísticas y econométricas para estimar aspectos del comportamiento económico de la empresa.

\section{Marco de Teoría}

Las técnicas de producción son las distintas maneras de obtener un producto. La forma específica como se combinan los factores productivos, el orden o la secuencia en que se utilizan, y el momento en que se utilizan dentro del proceso productivo es lo que se denomina la técnica de producción (Ballestero, 1983, p. 83).

\subsection{La función de costo total}

Nicholson (2007, p. 230) define la función de costo total señalando que "el costo total mínimo en que incurre la empresa, dados los precios de los factores y el nivel de producción es:

$C T=f\left(P X_{i^{\prime}} Q\right)^{\prime \prime}$

$\mathrm{CT}=$ costo total (en valores monetarios); $P X_{i}=$ precios de los insumos o factores; $i=1,2,3, \ldots, n ; Q=$ cantidad de producto; $f=$ la forma funcional que relaciona a las variables.

La función de costos que se considera en esta investigación es de corto plazo. Esta se define como "el costo mínimo necesario para conseguir un nivel dado de producción, ajustando únicamente los factores variables" (Varian, 1999, p. 362). Este supuesto se apoya en el siguiente razonamiento teórico y empírico: en la empresa agraria, un factor fijo es la cantidad de tierra, medida en hectáreas, considerada como capital, ya que ha sido intervenida para aumentar su productividad en la obtención de pastos, utilizado para alimentar los ganados a cebar. Si se considera el tamaño de la empresa, medido por la cantidad de hectáreas bajo producción, este factor permaneció fijo a lo largo del período de estudio. Y si el tamaño se mide por las contrataciones salariales, considerando el número de trabajadores fijos en la nómina, estas también han estado constantes. 
En cuanto a las relaciones entre el costo total a corto plazo y el nivel de la producción, Katz \& Rosen (1994, p. 282) señalan que "la curva del costo total debe tener una pendiente positiva" ya que para aumentar el nivel de producción debe aumentar la cantidad de factores contratados, y más factores cuestan más dinero.

Varian (1999, p.289-291) y Nicholson (2007, p. 232-233), establecen:

i. La curva de costo medio puede descender al principio debido a los costos fijos medios decrecientes, pero después aumenta debido a los costos variables medios crecientes.

ii. La curva de costo marginal atraviesa a la curva de costo medio y a la curva de costo medio variable en sus puntos mínimos.

iii. El costo marginal y el costo variable medio de la primera unidad de producción son iguales.

\subsection{Desempeño económico empresarial}

El cálculo de los costos en la unidad empresarial es clave pues la evaluación del desempeño económico de la misma sería imposible sin ello. Al deducirlos de los ingresos obtenidos por la venta del producto en el mercado, se obtiene el excedente económico, que constituye el objetivo de un empresario racional. Dependiendo de qué clases de costos se utilicen para descontar de los ingresos, las medidas del excedente económico pueden ser, de acuerdo con Ballestero (1991), las siguientes:

i. El margen bruto, se obtiene de deducir a los ingresos solamente los costos que implican desembolsos de dinero en efectivo, especialmente, los costos de fuerza de trabajo, los costos de capital circulante y otros servicios contratados por la empresa.

ii. El margen neto, se obtiene si se deducen de los ingresos, además de los costos anteriores, los costos de depreciación del capital fijo.

iii. El beneficio empresarial. $\mathrm{Si}$ a los ingresos se le deducen todos los costos anteriores más el costo de oportunidad, se obtiene el beneficio empresarial.

En esta investigación se calcula el margen bruto, tanto en valores absolutos como relativos, para evaluar el desempeño económico de la empresa.

\subsection{La oferta individual de la empresa}

La función de oferta de la empresa precio-aceptante, desde el punto de vista de la Microeconomía convencional, se obtiene de un problema de maximización de los beneficios. Como señala Mundlak (2001, p. 47): "analíticamente, la función de oferta de la empresa competitiva es la primera derivada parcial de la función de ganancia con respecto al precio del producto".

En un mercado competitivo, la curva de ofertaprecio se obtiene de igualar el ingreso marginal con el costo marginal para significar que un empresario producirá hasta el nivel de producto en el que el ingreso obtenido por la venta de una unidad adicional del mismo se iguala con el costo adicional en el que se incurrió para producirlo. Esto es, el ingreso marginal es igual al costo marginal. En los mercados competitivos, además, la variación del ingreso por vender una unidad más de producto en el mercado es equivalente al precio del producto.

Dicho por Varian (1999, p. 389) "en el nivel óptimo de producción, una empresa competitiva debe producir en el punto en que el precio es igual al costo marginal. Cualquiera que sea el nivel del precio del mercado, $P$, la empresa elegirá el nivel de producción, $Q$, en el que $P=C M(Q)$. Por lo tanto, la curva de costo marginal de una empresa competitiva es precisamente su curva de oferta". Esta relación funcional entre la oferta y el precio, lo demás constante, indica la cantidad máxima de producto que se está dispuesto a ofrecer a los distintos precios del mercado del producto en mención.

La evidencia empírica muestra que las empresas del sector agrario en Colombia se aproximan a ser precioaceptantes. Castillo, (2015) encontró índices de concentración de las ventas en el período 2009-2011 que oscilaron entre 16,5 y $20,4 \%$ en el $I C_{4}$; y entre 24,3 
y $29,5 \%$ en el $I C_{8}$. No parece existir, de acuerdo con Bain (citado por Caldentey, 1998, p. 72), y a la luz de tales indicadores, poderes de mercado.

\section{Metodología}

La información estadística fue obtenida de los registros históricos de la empresa Lituania, entre Noviembre/2005 a Enero/2014. Es de periodicidad cuatrimestral. Los costos que se consideran son los costos privados. El cálculo de los costos externos generados por la ganadería, especialmente los ambientales, está por fuera del alcance de este trabajo. Los costos variables están constituidos por los pagos en dinero a la fuerza de trabajo en labores de fumigación y desmonte de los potreros, la compra de terneros, que representan el insumo principal para la obtención del animal cebado, y servicios contratados. Los costos fijos, incluyen los pagos salariales a trabajadores fijos en la nómina, la compra de sal de ganado para consumo de los animales, los gastos de agua, luz y gas, la aplicación de vitaminas, vacunas y drogas veterinarias para la salud animal, la reparación y mantenimiento de cercas, combustible. Todos estos costos implicaron desembolsos de dinero en efectivo y son prácticamente irrecuperables cuando se toman decisiones posteriores de asignación de los recursos.

La medición de indicadores técnicos de productividad de la tierra, como la cantidad de kilos por hectárea por año, $w / h a$; la ganancia de peso por animal, y ganancia de peso por animal por día, gw/d, se han calculado así:

i) $\frac{w}{h a}=\left(\frac{W_{f}-W_{i}}{\# h a}\right)\left(\frac{12}{10}\right) . W f=$ peso final del novillo $(\mathrm{kg})$; $W t=$ peso inicial; 12 = \# de meses de ocupación de las paraderas; 10 = \# de meses de ocupación por un lote especifico de ganado; \# ha= número de hectáreas sembradas en pastos.

ii) $g w=\frac{W_{f}-W_{i}}{\# a} ; \# a=$ número de animales.

iii) $\frac{g w}{d}=\frac{\left(W_{f}-W_{i}\right) / \# a}{300} ; 300=\#$ de días de ceba

La medición del margen bruto en valores monetarios -que se utiliza como indicador de desempeño económico de la unidad productiva-, se hizo deduciendo de los ingresos los costos totales que supo- nen desembolsos de dinero en efectivo como los anotados antes.

Los ingresos se calcularon multiplicando la cantidad de kilos producidos y vendidos en pie por el precio corriente de venta en los tres cuatrimestres de un año. Los precios varían en sus niveles, pero mediante la realización de las pruebas de medias con el método Anova F test y Welch F test (Lilien et al., 2007, p. 395-396), se comprobó que no tienen diferencias estadísticas significativas entre y dentro de los pre$\operatorname{cios}^{1}$. Por ende, es indiferente, en términos estadísticos, utilizar una media constante u otro nivel.

Los indicadores de desempeño económico relativo, en particular el margen bruto por unidad de producto final, y el margen bruto sobre costos, se calcularon así:

$M B U=\frac{M B}{Q}$

$M B U=$ margen bruto unitario; $Q=$ cantidad de kilos producidos.

El margen bruto sobre costo se calcula:

$M B C=(M B / C) \times 100$

$M B C=$ margen bruto sobre costos.

La función de costo total, bajo las consideraciones hechas en el marco teórico, se estimó utilizando el método de mínimo cuadrados ordinarios.

$C T_{t}=f\left(Q_{t}, P c_{t}, \varepsilon_{t}\right)$

$C T=$ costo total (en valores constantes del año 2006, deflactados por el índice de precios al productor para el sector agrario, según DANE, base diciembre 2006); $Q$ = cantidad de kilogramos de ganado vivo vendidos; $P c=$ precio del principal insumo: los terneros de levante; $\varepsilon_{t}=$ error aleatorio que recoje la influencia de las variables desconocidas, con características de media cero, varianza constante y no autocorrelación.

1 La prueba Anova F test aceptó la hipótesis de igualdad de medias de los precios al 4\%; la Welch $\mathrm{F}$ test al $5 \%$ de significación. 
Variaciones de $Q$ afectan a $C T$ pues supone utilizar más (o menos) cantidades de insumos, que cuestan; pero variaciones de $C T$ por el uso de más insumos (o menos) también pueden afectar a $Q$, por ende podría darse un problema de variable explicativa endógena (Kennedy, 1997) que hace inconsistente la estimación mínimo- cuadrática de los parámetros de la ecuación (4). Para descartar tal posibilidad de aplicó la prueba de Hausman (1978) probando que no existe correlación contemporánea entre variables que afectan a $Q$, contenidas en un término de error, $\mu_{t}$, y los regresores de CT.

La estrategia de la prueba consiste en:

i. Estimar $Q_{t}=f\left(L_{t}, \mu_{t}\right)$, donde $L$ es el número de animales de levante comprados; $\mu_{t}$ es un término de error.

ii. Obtener la cantidad de producto obtenido estimado, $\widehat{Q}_{f}$, y los residuos, $\pi_{\mathrm{t}}$, de esa ecuación.

iii. Estimar $C T_{t}=f\left(P_{c}, \hat{Q}_{f}, \mu_{t}\right)$.

iv. Si $\mu_{t}$ no es significativo en esta estimación de $C T$, significa que no hay problemas de endogeneidad entre $C T$ y $Q$.

Para asegurar que el error, $\varepsilon_{t}$, de la ecuación (4) tenga las características estadísticas deseables de la teoría clásica de regresión lineal, se evaluó que los mismos no estuviesen afectados por correlación serial y varianza heterocedastica. La detección de presencia de correlación serial de primer orden se realizó mediante la prueba de Durbin-Watson, D-W. (Lilien et al., 2007, p. 64-65).

Además, aunque la muestra es pequeña, se utilizó la prueba multiplicador de Lagrange, LM, de correlación serial para detectar la presencia de retardos mayores. Esta contrasta la hipótesis nula: no hay correlación serial en los residuos hasta el orden especificado. Dos estadísticos, el llamado estadístico F y el $N \times R^{2}$ (el número de observaciones por el $\mathrm{R}$ cuadrado) con distribución Chicuadrado, se usa para tomar la decisión (Lilien et al., 2007, p. 65).
Para evaluar la existencia de varianza constante se acudió a la prueba de White (1980). Esta examina si la varianza del error se ve afectada por cualquiera de los regresores, sus cuadrados o sus productos cruzados.

Asumiendo constante el precio del insumo, se obtuvo la función de costo medio y costo marginal en función de la cantidad de producto. La obtención de la ecuación y la curva de oferta-precio provienen de igualar el costo marginal de la empresa con el precio real del producto en el mercado de ganado vivo en el departamento de Córdoba.

Se utilizó el software Econometric Views 6.0, Lilien et al., (2007) para los cálculos econométricos.

\section{Resultados}

\subsection{Técnica de producción}

Distintas técnicas se utilizan para producir ganado de ceba. En particular, la de la empresa Lituania se describe en la tabla 1, teniendo en cuenta el orden o la secuencia del proceso de la ceba. Se definen las distintas etapas, las actividades, los insumos que intervienen y las cantidades utilizadas.

La primera etapa es la preproducción. Esta tiene que ver con la adecuación de terrenos para siembra y conservación de los pastos. Comprende las actividades de fumigación contra las malezas de los potreros y se indican en la tabla 1 desde el insumo número 1 al 7, según el orden en el que se emplean. Las actividades de arreglo de cercas en los corrales y potreros comprenden desde el insumo 8 al 14. La actividad del tratamiento del agua para suministrar a los animales va desde el insumo 15 hasta el 19. La actividad de abonamiento de la tierra comprende los insumos marcados con el número 20 al 22. La actividad de compra de ganado se hace en finca o en las subastas ganaderas. Comprende los insumos marcados con el número del 23 al 26.

La segunda etapa es la de producción. Consiste en el proceso de suministrar a los animales alimenta- 
Tabla 1. Técnica de producción del ganado de ceba en la empresa Lituania

\begin{tabular}{|c|c|c|c|c|}
\hline Insumo & Cantidad & Precio (pesos) & Insumo & Cantidad \\
\hline 1. Combatrán & 1 ca (20 litros) & 450.000 & 21. Trabajador & 2 \\
\hline 2. Glifosato & 1 (20 litros) & 170.000 & 22. Caballos & 2 \\
\hline 3. Esterón 47 & 5 galones & 48.000/galón & 23. Ganados & $50-70$ \\
\hline 4. Plenun & 1 caneca & 800.000 & 24. Básculas & 1 \\
\hline 5. Agua & $20 \mathrm{lt}$ / dosis & & 25. Corral & 1 \\
\hline 6. Bombas & 04-jun & & 26. Camiones & \\
\hline \multirow{2}{*}{ 7. Trabajadores } & \multirow{2}{*}{ 04-jun } & $12.000 /$ & \multirow{2}{*}{ 27. Enterogan (vit) } & \multirow{2}{*}{$1 \mathrm{cc} / 50 \mathrm{k}$} \\
\hline & & trabajador & & \\
\hline 8. Astillas & 40/hectárea & & 28. Next platino (purgante) & $1 \mathrm{cc} / 50 \mathrm{k}$ \\
\hline 9. Alambre & $1 \mathrm{rollo}=800 \mathrm{mt}$ & & 29. Figabol (anabol & $1 \mathrm{cc} / 90 \mathrm{k}$ \\
\hline 10. Aisladores & 80 & & 30. Combibac (vac) & $5 c c$ \\
\hline 11. Guadañas & 1 & & 31. Ganabaño & 1 litro \\
\hline 12. Gasolina & 1 galón & & 32. Trabajadores & 3 \\
\hline 13. Caballos & 2 & & 33. Bombas & \\
\hline 14. Trabajadores & 3 & & 34. Sal & 5 bultos \\
\hline 15. Cloro & 41 ts & & 35. Caballos & 2 \\
\hline 16. Electrobomba & 1 & & 36. Trabajador & 3 \\
\hline 17. Tanque & 1 & & 37. Ingeniero agrónomo & \\
\hline 18. Pozo art & 1 & & 38. Corral & \\
\hline 19. Alberca & 4 & & 39. Bascula & \\
\hline 20. Urea & 2 bulto de 50 kg & & 40. Camiones & \\
\hline
\end{tabular}

Fuente: Elaboración de los autores con base en información de la empresa Lituania.

ción, vitaminas y vacunas durante un período de 10 a 12 meses para alcanzar un peso ideal entre 450 - 500 kilos en pie. Comprende las actividades de alimentación complementaria, vacunación y baño de los animales; los insumos utilizados van desde 27 hasta 33. La actividad de ceba y rotación comprende el suministro de sal diariamente; cada tres meses los animales se bañan y vacunan. Los insumos comprenden desde 34 al 37. El proceso de rotación consiste en rotar los animales al siguiente potrero entre 5 a 7 días dependiendo la cantidad de pasto que haya en el potrero. Se hace por medio de módulos, cada módulo consta de unas rotaciones, las cuales tienen una extensión de 3 hectáreas.

La etapa tercera es la de comercialización, consiste en la venta de los animales, la cual se hace en la finca o en el frigorífico. Comprende los insumos 38 al 40.

Con esta técnica se obtuvieron los resultados técnicos que se muestran en la tabla 2. 
Tabla 2. Indicadores técnicos de la empresa Lituania, 2006-2013

\begin{tabular}{|l|c|c|c|c|}
\hline \multicolumn{1}{|c|}{ Años } & $\begin{array}{c}\text { w/ha(kg/ } \\
\text { ha/a) }\end{array}$ & gw (kg/a) & $\begin{array}{c}\text { Nro } \\
\text { animales }\end{array}$ & $\begin{array}{c}\mathbf{g w} / \mathbf{d} \\
\mathbf{( k g / a / d )}\end{array}$ \\
\hline 2006 & 518 & 213 & 170 & 0,7 \\
\hline 2007 & 550 & 197 & 195 & 0,7 \\
\hline 2008 & 536 & 208 & 180 & 0,7 \\
\hline 2009 & 523 & 209 & 175 & 0,7 \\
\hline 2010 & 432 & 202 & 150 & 0,7 \\
\hline 2011 & 484 & 218 & 186 & 0,7 \\
\hline 2012 & 541 & 240 & 189 & 0,8 \\
\hline 2013 & 446 & 217 & 172 & 0,7 \\
\hline media & 504 & 213 & 177 & \\
\hline desv tipica & 42 & 12 & 13 & \\
\hline coef variac & 0,083126793 & 0,05684068 & 0,073743133 & \\
\hline
\end{tabular}

Fuente: Cálculos del estudio con base en información de la empresa Lituania.

Kilogramos obtenidos por hectárea por año: osciló entre 432 y 550 con un coeficiente de variación del $8,3 \%$. Ganancia de kilogramos por animal varió entre 197 y 240 con coeficiente de variación del $5,7 \%$. Número de animales por hectárea, osciló entre 2,1 y 2,4 .

El sistema de información de precios del sector agrarios, SIPSA, reportó para actividad de ceba de 11 meses en unidades empresariales de 50 a -500 animales en la Costa Caribe ganancias de peso por animal de 243 kilos en el ciclo de producción (SIPSA, 2010). Corpoica (2002) en la región Caribe colombiana señala que los sistemas de alimentación basados en pastoreo de forrajes tropicales y el uso de alimentos complementarios y sal mineralizada permiten cargas animales que oscilan entre 1,9 a 2,5 unidades de animal por hectárea; mayores ganancias de peso por animal y por día, y mayor producción de carne por hectárea por año.

\subsection{El desempeño económico}

En este numeral se presentan los resultados económicos de la empresa ganadera de ceba Lituania durante el período 2005-2014 en términos de los ingresos obtenidos por la venta de su principal producto: animales cebados en pie, y de los costos en los que se incurre para producirlos. De los ingresos se han deducido los costos para calcular el excedente económico. Puesto que los costos se han considerado solamente aquellos que implicaron desembolsos de dinero en efectivo y no se consideró la depreciación del capital fijo, el indicador de excedente económico que se calculó es el de un margen bruto.

Tabla 3. Indicadores de desempeño económico de la empresa ganadera Lituania, 2005-2014.Valores monetarios en millones de pesos corrientes

\begin{tabular}{|c|c|c|c|c|c|c|}
\hline \multirow{3}{*}{ Años } & \multirow{3}{*}{ Ingresos } & \multirow{3}{*}{ Costos } & $M B=$ & $\mathrm{MBC}=$ & $\begin{array}{c}\mathrm{Kg} \\
\text { vendidos } \\
\end{array}$ & \multirow{3}{*}{$\begin{array}{l}\text { MBU } \\
(\$ / \mathrm{kg})\end{array}$} \\
\hline & & & $\begin{array}{c}\text { Margen } \\
\text { bruto total }\end{array}$ & $\begin{array}{c}\mathrm{MB} / \\
\text { costos }\end{array}$ & (miles) & \\
\hline & & & & $(\%)$ & & \\
\hline 2006 & 241,5 & 143,5 & 98 & 68,3 & 83,62 & 1.172 \\
\hline 2007 & 261,3 & 155,3 & 106 & 68,2 & 92,91 & 1.141 \\
\hline 2008 & 250,7 & 151,1 & 99,6 & 65,9 & 89,79 & 1.109 \\
\hline 2009 & 223,7 & 139,3 & 84,4 & 60,6 & 85,18 & 991 \\
\hline 2010 & 173,8 & 148,2 & 25,6 & 17,3 & 73,65 & 348 \\
\hline 2011 & 232,2 & 169,6 & 62,6 & 36,9 & 90,78 & 690 \\
\hline 2012 & 253,5 & 157,9 & 95,6 & 60,5 & 95,23 & 1.004 \\
\hline 2013 & 220,6 & 187,1 & 33,5 & 17,9 & 83,15 & 403 \\
\hline
\end{tabular}

Fuente: Cálculos de los autores con base en información de la empresa Lituania.

La capacidad de producción de carne en pie osciló durante el período de estudio entre 73.650 kilogramos en el año 2010 y 95.232 kilogramos anuales en el año 2012. Los ingresos variaron desde un máximo de $\$ 261,3$ millones de pesos corrientes en el año 2007 a un mínimo de $\$ 173,8$ millones en el año 2010, que fue el año del nivel de precio más bajo y de la menor cantidad producida.

De los ingresos, se destaca su amplia variabilidad. $\mathrm{Si}$ esta se mide por el coeficiente de variación, $\mathrm{CV}$, 
es alto: $28,9 \%$. Es un indicativo del riesgo económico del negocio ganadero, el cual está afectado, principalmente por las variaciones de las condiciones climáticas y la variabilidad del precio del producto en el mercado. En la región del valle del rio Sinú existen dos épocas bien marcadas de precipitaciones pluviales: una época de lluvia entre mayo/ noviembre con pastos abundantes y frescos que mejoran la condición física de los animales; y una época de sequía entre noviembre/abril con escasez de pasturas naturales que desmejoran la condición física de los animales. Existe evidencia empírica que demuestra que estacionalmente el precio del ganado en pie aumenta en la época de lluvia y desciende en la época seca (Castillo, 2009, p. 57). De esta forma, la época de venta afecta los ingresos del productor, especialmente si no hay contratos de precios de venta a mediano plazo.

Adicionalmente, se ha calculado el coeficiente de correlación lineal de Spearman para examinar la relación del ingreso con el precio y las cantidades. El resultado indica que las variaciones del ingreso se relacionan positiva y significativamente con las variaciones de las cantidades de kilogramos vendidas con un coeficiente de correlación del 0,99. Las variaciones del ingreso con las variaciones del precio no fueron significativas pues se aceptó la hipótesis nula de que el coeficiente de correlación es cero. Este resultado sería otra confirmación que el empresario ajusta los ingresos modificando las cantidades; ya que el precio se lo determina el mercado.

El margen bruto total, en valores absolutos, osciló entre \$106,0 millones de pesos en el año 2007 y $\$ 25,6$ millones en el 2010. El margen bruto sobre costos fue más alto en los años 2006-2009, aunque decreció de $68,3 \%$ a $60,6 \%$. Esto significa que por cada 100 pesos invertidos se obtuvo un excedente sobre el costo de $\$ 68,3$ y $\$ 60,6$ pesos. Este margen se redujo entre 2010-2011 cuando cayó a 17,3\% y $36,99 \%$ respectivamente. El margen bruto sobre costos se volvió a recuperar en el 2012 y se redujo a niveles del 17,9\% en el año 2013.

Para unidades productivas medianas de ceba de 11 meses en la Costa Caribe, el Observatorio de precios y costos agrarios del noroccidente del Caribe colom- biano, OPCA, encontró durante el 2010 márgenes brutos medios entre 54,0 y 29,5\% (OPCA, 2011).

El margen bruto por kilogramo vendido, siguió igual tendencia del indicador anterior. Estuvo entre $\$ 1.172$ y $\$ 991$ pesos por cada kilogramo vendido entre 2006-2009; se redujo a \$348 en el 2010; se recuperó entre 2011-2012 y cayó en 2013 a \$403 pesos por kilogramo. Delgado et al. (2008) en Brasil, India, Filipinas y Tailandia probaron que los productores en pequeña escala obtienen ganancias por unidad de producto mayores o iguales que las de los grandes productores.

\subsection{La función de costos y de oferta -precio del ganado de ceba}

En esta parte se utilizan elementos de la teoría de la regresión de series de tiempo para estimar la función de costos totales en el proceso de producción de carne de ganado bovino en pie en la empresa Lituania. Igualmente, se estima la función de oferta -precio a partir de la curva de costo marginal estimada y del precio medio del mercado prevalente en la región durante el período de estudio.

\subsubsection{La función de costo total}

La función de costos estimada es de la forma:

$C T_{t}=\beta_{0}+\beta_{1} Q_{t}^{2}+\beta_{3} P c_{t}+\varepsilon_{t}$

$C T=$ costo total (pesos constantes del 2006); $Q=$ cantidad producida de carne de ganado vacuno en pie, en kilogramos; $P c=$ precio de los terneros de levante; $\beta_{0}, \beta_{1}, \beta_{2}$ son parámetros a estimar; $\varepsilon=$ término de error aleatorio; $t=$ índice de tiempo desde noviembre 2005 a enero 2014.

El desarrollo de la prueba de Hausman indicó que el valor de $\pi t$ no es significativamente diferente de cero, lo que significa que no hay problemas de variable explicativa endógena, esto es, $Q$ es exógena a $C T$; por tanto, la estimación mínimo- cuadrática de los parámetros es consistente ${ }^{2}$.

2 Por razones de espacio no se incorporan las pruebas, pero están disponibles para los editores y lectores que lo requieran. 
En la tabla 4 se muestra la ecuación de costo total estimado: los parámetros, la significatividad estadística de los mismos, y el coeficiente de determinación ajustado. El término $\beta_{0}$ representa la constante del modelo y se interpreta como el costo fijo en que incurre la unidad productiva en el proceso de producción. Es significativo al 1\%. Es decir, se rechaza la hipótesis nula que sea cero con un nivel de confianza del 99\%.

Tabla 4. Modelo de costos totales de la empresa Lituania

\begin{tabular}{|c|r|r|r|r|}
\hline Variables & Coeficiente & $\begin{array}{c}\text { Error } \\
\text { estándar }\end{array}$ & Estadístico $\boldsymbol{t}$ & Probabilidad \\
\hline$\beta_{0}$ & 23.997 .668 & 5.467 .332 & 4,39 & 0,0000 \\
\hline$Q^{2}$ & 0,02135 & 0,001 & 15,36 & 0,00000 \\
\hline$P c$ & 19,8054 & 8,25 & 2,4 & 0,0248 \\
\hline
\end{tabular}

Fuente: Elaboración propia.

El R ${ }^{2}$ Ajustado fue de 0,937. El parámetro $\beta_{1}$, se interpreta como el impacto que tiene sobre los costos un salto de la producción en una potencia 2. Así, un salto de la producción desde 10 a 100 kilos de carne aumenta el costo total en 2,1 pesos. Este parámetro es significativo al $1 \%$.

El parámetro $\beta_{2}$, señala el impacto del precio del insumo terneros de levante sobre el costo total. Indica que una variación de una unidad monetaria produce una variación del costo en 19,8 unidades monetarias. Es significativo al 5\%.

Este modelo uniecuacional econométrico no tiene problemas de autocorrelación. La prueba multiplicador de Lagrange, LM, hasta el segundo retardo permite aceptar al 5\% la hipótesis nula de no autocorrelación con el estadístico F, y con el del número de observaciones multiplicado por el $\mathrm{R}$ cuadrado.

Tampoco tiene problemas de heterocedasticidad. La prueba de White permite aceptar la hipótesis nula de homocedasticidad al $5 \%$.

Las variables consideradas en el modelo explican el $93,7 \%$ de las variaciones del costo total, lo cual es alto.

\subsubsection{La función de costo medio y costo margi- nal en función del producto}

Más que la función total de costos, interesa la función de costo medio y marginal, las cuales indican el comportamiento de los costos por unidad de producto, $y$ permiten comparar los costos unitarios con el precio unitario del producto final al venderse. Derivando parcialmente la ecuación (4A), se construyen tales funciones. Considerando constante el precio del insumo, la función de costo medio es:

$$
C M=\frac{23.997 .768}{Q}+0,021346 Q
$$

Bajo la misma condición, la función de costo marginal es:

$$
\frac{\partial C T}{\partial Q}=2 \times 0,021346 Q=0,042692 Q
$$

La ecuación (6) indica que un incremento (disminución) en la producción en 100 kilogramos adicionales aumenta (disminuye) el costo total en $\$ 4,27$ pesos constantes de diciembre del 2006. Por lo tanto, la producción de ganado de carne en la empresa bajo análisis tiene costo marginal creciente, al menos en el tramo de la producción cuatrimestral entre 17.649 kilos y $48.780^{3}$. Los factores determinantes de ello están por fuera de los objetivos de este trabajo y deberían abordarse en temas de investigación posteriores. La evidencia empírica internacional, recogida en el trabajo de Chavas (2001) indica que en la agricultura coexisten unidades productivas con costos medios y marginales crecientes, decrecientes y/o constantes dependiendo del tamaño y del tipo de tecnología.

El costo medio por kilogramo osciló entre $\$ 1.431$ y $\$ 1.736$ (pesos constantes del año 2006). El Observatorio de precios y costos agrarios del noroccidente del Caribe colombiano, OPCA, (2011) ha reportado con base en información del sistema de información de precios del sector agrario, SIPSA, costos medios por kilo en el Caribe colombiano

La comprobación matemática de ello es que la segunda derivada de la función de costo total con respecto a $\mathrm{Q}$, equivalente a la pendiente de la función de costo marginal, es positiva. 
oscilantes entre $\$ 1.785$ y $\$ 2.035$ en el año 2010 en empresas de ganadería de ceba medianas (entre 50 y 500 animales). Convertidos a pesos reales de 2006 equivalen a costos promedios de $\$ 1.503$ y 1.714 por kilo, respectivamente.

Igualando la función (5) y (6) se encuentra que la curva de costo marginal intersecta a la curva de costo medio cuando se producen 33.529 kilogramos de ganado vivo. El costo medio mínimo de la empresa en este nivel de producción corresponde a $\$ 1.431$ pesos constantes del año 2006 por kilogramo de ganado vivo. Este punto de costo mínimo refleja la escala mínima eficiente del proceso de producción examinado, como lo indica teóricamente Nicholson (2007, p. 232). Además, este hallazgo empírico indica que un precio de mercado por kilogramo de carne por debajo de $\$ 1.431$ pesos, es decir, por debajo del costo medio por kilo, señalaría el punto de cierre de largo plazo de la empresa.

\subsubsection{Función de oferta-precio de carne de ga- nado bovino en pie}

En este numeral se construye la curva de oferta- precio de la producción de carne de ganado vacuno en pie en la empresa igualando la función de costo marginal con el precio real prevaleciente en el mercado. Como ya se ha señalado, el mercado de ganado vacuno en pie opera como vendedor en condiciones competitivas o cercanas a ellas. En consecuencia, el costo marginal es igual al ingreso marginal, equivalente al precio del producto, en un mercado de tales características. La función de oferta que se obtiene es parcial ya que solamente explica las variaciones de las cantidades producidas $u$ ofrecidas en función del precio del producto en el mercado y se consideran constantes otros factores determinantes de la oferta, tales como el precio de los insumos, el precio o la rentabilidad de otros productos, la tecnología, y el riesgo precio y el riesgo rendimiento (Tomek \& Robinson, 2004).

Igualando la función de costo marginal (6) al precio de venta en el mercado de ganado vivo, en Córdoba, se tiene que la función de oferta- precio de la empresa:
$Q_{O}=23,42 P_{0}$, ceteris paribus.

La ecuación (7) es una función de oferta -precio lineal $e$ indica que, ceteris paribus, un incremento (disminución) de 1 una unidad monetaria en el precio del ganado cebado vacuno en pie provoca un aumento (disminución) de 23,42 kilogramos de carne de ganado vivo en la empresa. La pendiente positiva de la función es expresión de la existencia de costos marginales crecientes. La literatura internacional sobre la producción y la oferta agraria, resumida por Mundlak (2000, 2001), señala la respuesta positiva de los agricultores a los incentivos de precios.

\section{Conclusiones y recomendaciones}

En este trabajo se analizó la producción de ganado bovino de carne en la unidad productiva Lituania en el departamento de Córdoba, Colombia, durante el periodo 2005-2014. Los resultados arrojan evidencia empírica adicional acerca de las características del comportamiento económico predicho por la teoría microeconómica convencional en unidades empresariales que tienen como objetivo la obtención de excedentes económicos y operan en condiciones de mercado cercanas al modelo competitivo básico. En particular se confirma:

i. El uso de técnicas de producción de ganado de carne intensivas en el uso de pasturas naturales mejoradas, complementos alimenticios y cuidado de la salud animal permite obtener resultados técnicos, en términos de producción y productividad, compatibles con la generación de excedentes económicos positivos.

ii. Desde el punto de vista económico, los ingresos tienen una alta variabilidad. Ello es una expresión del riesgo en esta actividad. El riesgo técnico procede, básicamente, de las condiciones ambientales ocasionado por la existencia de dos épocas bien diferenciadas de precipitaciones: una lluviosa, de mayo a octubre; y otra seca, de noviembre a abril. Estas dos épocas afectan la alimentación de los animales la cual se hace predominantemente con pasturas naturales, por ende, afectan también la condición y desarrollo físico del animal. Estas condiciones 
climáticas también pueden ser un factor que ocasiona enfermedades en los animales si no se tiene un control sanitario riguroso. El riesgo económico procede de las variaciones del precio como consecuencia que las condiciones climáticas producen estacionalidad en la producción y en los precios. Estos últimos tienden al alza en la época de lluvia, y a la baja en la época de sequía.

iii. La empresa tiene márgenes brutos positivos en el desarrollo de su actividad. El margen bruto sobre costos es comparable con los márgenes existentes en la región Caribe colombiana para unidades de producción medianas.

iv. La función de costo total mantiene una relación positiva con la producción y el precio de los insumos. El costo medio desciende con la producción hasta el nivel de 33.529 kilogramos de ganado vivo. En este punto alcanza un costo mínimo de $\$ 1.431$ pesos por kilogramo, posteriormente asciende. El costo marginal es creciente. En concreto, la información empírica disponible indica que un incremento de 100 kilogramo adicional en la cantidad de producto aumenta el costo marginal en $\$ 4,3$ pesos constantes del 2006.

v. En consonancia con la existencia de costo marginal creciente, la empresa tiene una función de oferta-precio con pendiente positiva. La función inversa de oferta indica que para que la empresa aumente la producción de ganado vivo en 1.000 kilogramos debe compensársele con un aumento en el precio de mercado de $\$ 47$ pesos constantes del 2011.

Estos resultados confirman que los productores de ganado responden a los incentivos de precio en la producción. Sin embargo, con el fin de evaluar con juicios más severos el desempeño económico de la empresa se recomienda adoptar un sistema más riguroso de información sobre precios y cantidades compradas y usadas de los distintos factores $e$ insumos que se utilizan en la técnica de ceba, los costos de depreciación del capital, y otros, con el fin de avanzar hacia la construcción de la función de producción, de la función de costo mínimo, de los efectos de los cambios de precios de los factores en la intensidad del uso de los mismos, y la función de oferta total.

Igualmente, sería deseable que para aminorar el riesgo de las transacciones de mercado la empresa adoptara con sus proveedores de ganado de levante y con sus compradores del producto final contratos a medio plazo.

\section{Referencias}

Ballestero, E. (1983). Principios de la Economía de la Empresa. Madrid, España: Alianza Universidad textos.

Ballestero, E. (1991). Economía de la Empresa Agraria y Alimentaria. Madrid, España: Mundiprensa.

Caldentey, P. (1998). Nueva Economía Agroalimentaria. Madrid, España: Editorial agrícola española.

Castillo, O. (2009). Mercados y Precios del Ganado en el Noroccidente del Caribe Colombiano. Montería, Colombia: Universidad de Córdoba.

Castillo, O. (2015). Economía Agraria: Apuntes de clase. Montería, Colombia: Universidad de Córdoba.

Chavas, J. P. (2001). Structural Change in Agricultural Production: Economics, Technology and Policy. En, B. Gardner and G. Rausser (Eds) Handbook of Agricultural Economics. Volume 1A, Chapter 5, pp 263-285. North Holland, Amsterdam: Elsevier Science.

Corpoica (2002). Producción de carne bovina de alta calidad en Colombia. Recuperado de : http://www.corpoica.org.co/sitioweb/ Archivos/Publicaciones/ProduccindecarnebovinadealtacalidadenColombia.pdf (visitada el 20/7/2014).

Delgado, C., Narrod, C. \& Tiongeo, M. (2008). Determinants and Implications of the Growing scale of Livestock Farms in Four Fast -Growing Developing Countries. Recuperado de: http:// www.ifpri.org/sites/default/files/pubs/pubs/abstract/157/rr157. pdf (visitada el 20/7/2014).

DANE (2013). Encuesta Nacional Agropecuaria 2013. Recuperado de: https:/www.dane.gov.co/index.php/agropecuario/encuestanacional-agropecuaria (consultada 20/7/2014).

Hausman, J. (1978). Specification Test in Econometrics. Econometrica, vol. 46, pp 1251-1271.

Kennedy, P. (1997). Introducción a la Econometría. FCE. México

Lilien, D. et al. (2007). Econometric- views versión 6.0, User's Guide II, Quantitative, USA: Micro Software.

Katz, M. \& Resen, H. (1994). Microeconomía. USA: Adison-Weslwy Iberoamericana.

Mundlak, J. (2000). Agriculture and Economic Growth. Theory and Measureament. England: Harvard University Press.

Mundlak, J. (2001). Production and Supply. En: B. Gardner \& G. Rausser (Eds): Handbook of Agricultural Economics, Volume 1A, Chapter 1: 3-85. North Holland: Elsevier science.

Nicholson, W. (2007). Teoría Microeconómica. España: Mc Graw Hill, España.

OPCA (2011). Comportamiento del índice de precios al productor de ganado vacuno en pie en Córdoba, Colombia, Junio 2011. 
Recuperado de: http://web.www3.unicordoba.edu.co/sites/ default/files/Indice\%20de\%20precio\%20al\%20productor\%20 de\%20ganado\%20en\%20Cordoba\%2Cjun\%202011.pdf (Fecha de consulta, 5/7/2014).

SIPSA (2010). Costos de producción de carne bovina. Recuperado en:http://www.agronet.gov.co/www/htm3b/public/boletines/ Costos2010trim1/costos2010T1_archivos/frame.htm (consultado 5/2014).
Tomek, W. \& Robinson, K. (2004). Agricultural Product Prices. USA: Cornell University Press.

Varian, H. (1999). Microeconomía Intermedia. Barcelona, España: Antoni Bosch.

White, H. (1980). A Heteroskedasticity-consistent Covariance Matrix Estimator and a Direct Test for Heteroskedasticity. Econometrica, 48, 817-838. 